\title{
An Effective Load Balancing Task Allocation Algorithm using Task Clustering
}

\author{
Poornima Bhardwaj \\ Research Scholar, Department \\ of Computer Science \\ Gurukul Kangri \\ Vishwavidyalaya,Haridwar, \\ India
}

\author{
Vinod Kumar, Ph.d \\ Professor \& Head, Department \\ of Computer Science, Gurukul \\ Kangri Vishwavidyalaya, \\ Haridwar, India
}

\begin{abstract}
In Distributed Computing Systems (DCSs), a program is split into small tasks and distributed among several computing elements to minimize the overall system cost. Several challenges have been posed by this mode of processing which can be classified mainly into two broad categories. One class belongs to the hardware oriented issues of building such systems more and more effective while the other aims at designing efficient algorithms to make the best use of the technology in hand. The task allocation problem in a DCS belongs to the later class. Intrinsically, task allocation problem is NP- hard. To overcome this issue, it is necessary to introduce heuristics for generating near optimal solution to the given problem.
\end{abstract}

This paper deals with the problem of task allocation in DCSs in such a way that the load on each processing node is almost balanced. Further, the development of an effective algorithm for allocating ' $m$ ' tasks to ' $n$ ' processors of a given distributed system using task clustering by taking both Inter Task Communication Cost (ITCC) and the Execution Cost (EC) is taken into consideration.

\section{General Terms}

Distributed Systems, Task Allocation

\section{Keywords}

Distributed Computing Systems, Task Allocation, Static Load Balancing, Execution Cost, Communication Cost.

\section{INTRODUCTION}

The potential of distributed computing exists whenever there are several computers unified in some manner so that a program or a procedure running on one machine can transfer control to a procedure running on another. Distributed computers can be best exemplified as geographically dispersed logically and physically independent processors with decentralized system - wide control on resources and cooperative computation of programs. The task allocation problem is a fundamental aspect of distributed computing. It arises whenever the procedures or modules of a program are distributed over several interconnected computers and the program is active among processors as execution proceeds. The allocation problem deals with the question of allocating modules to the processors so as to minimize the cost of running a program [2].

The task allocation problem has multiple dimensions. Various aspects of the task allocation problem in a DCS have been covered by researchers. This problem has been tackled with various techniques through different allocation schemes considering different aspects such as inter- processor communication, reliability, load on the processors, memory utilization, etc. The objective of the present study has been to allocate tasks, constituting a distributed application, to available processing elements to optimize one or more measure(s) of effectiveness i.e. equalizing load on the processors, minimization of inter - processor communication (IPC), maximization of system reliability and minimization of total cost of execution etc. [16].

In a DCS, an allocation policy may be either static or dynamic, depending upon the time at which the allocation decisions are made. In a static task allocation, the information regarding the tasks and processor attributes is assumed to be known in advance.

System performance can be improved by transferring work from nodes that are heavily loaded to nodes that are lightly loaded. One of the objectives of using load balancing is to minimize the execution cost of the application. Efficient load balancing method can increase performance. Load - balancing algorithms can be generally classified as centralized or decentralized, dynamic or static, periodic or non-periodic, and those with thresholds or without thresholds [1]. In this work Static Load Balancing (SLB) is used. In SLB, the decision of assignment of tasks to processors is taken before the commencement of execution. Expected execution time and resource requirements are known a priori. Task is executed on the processors to which it is assigned at the beginning of execution as there is no task migration in SLB. The main purpose of the static scheduling is to minimize execution time and communication overhead [16, 17].

The heuristic-based scheduling techniques are the most common approaches for task scheduling. These are usually classified into three classes,

$-\quad$ Priority-based scheduling,
- $\quad$ Duplication-based scheduling and
Cluster-based scheduling

In priority-based scheduling, priorities are calculated and assigned to the tasks which are then scheduled onto processors according to the priorities. In duplication - based scheduling, while tasks are allocated to a processor, its parent (and predecessor) tasks are duplicated to occupy the idle times of the processor to eliminate the communication delay that occurs when message is passed from the parent tasks to the allotted task.

In cluster-based scheduling, some tasks, that need to communicate among themselves, are grouped together to form a cluster. Each of these tasks - clusters is then scheduled onto an available processor. The main problem arises when the number of clusters is more than the number of available 
processors. This leads to the scheduling of more than one cluster onto the same processor and unavoidably increases the overall schedule length. Approaches, that are used to minimize the total sum of execution and communication costs, are discussed in $[3,7,13,14,15$,

In this paper, a task allocation algorithm based on clustering, that finds a near optimal solution to the problem, is proposed. The developed algorithm tries to minimize the total system cost by forming cluster of tasks in such a way that the cluster, having minimum execution cost, is allocated first. The communication cost of the tasks in the same cluster is assumed to be zero. Using this strategy it has been observed that the total system cost is less as compared to that which is obtained by the heuristic reported in [5]

\section{RELATED WORK}

The basic idea of clustering based algorithm is to group heavily communicating tasks into the same cluster. The tasks that are grouped into the same cluster are assigned to the same processor in an effort to avoid communication costs. There are basically two types of clusters; linear and nonlinear. Two tasks are called independent if there are no dependent paths between them. A cluster is called nonlinear if there are at least two independent tasks in the same cluster, otherwise, it is called linear.

A class of directed acyclic graph scheduling algorithms is based on clustering $[4,8,20]$. The work done by Liou et al. indicates that if task clustering is performed prior to scheduling, the clusters are assigned to the processors in a balanced way, giving the benefit of load balancing [10]. Similar work is done by Palis et al. [11] to reduce the overall execution cost by using a simple greedy algorithm for task clustering. In the task clustering approach, proposed by Vidyarthi et al. [19], task modules are clustered on the basis of their communication overhead. Modules, having high communication overhead, are clustered to decrease the communication delays. The approach of clustering the heavily communicating tasks is used in [8, 17]. In [8], the authors choose those modules for clustering which are maximally linked from the point of view of the processor on which the assignment is to be done. Sharma et al. in [17] formed clusters on the basis of their communication cost to reduce the communication delays. In the work, done by Raii et al. [12], the total number of tasks in a cluster is not more than the total number of tasks divided by the total number of processors. Initially, all tasks are considered to be in a single cluster and stored in an array. The tasks, from this array, are selected for merging to form various clusters.

\section{PROBLEM FORMULATION}

The problem, being addressed in this paper, is concerned with an optimal allocation of the task - cluster of an application on the processors in a DCS. An optimal allocation is considered as one that minimizes the system cost function subject to the system constraints.

\subsection{Problem Statement}

Let the given DCS consist of a set of $n$ processors $P=\left\{p_{1}\right.$, $\left.\mathrm{p}_{2}, \ldots \ldots \ldots \mathrm{p}_{\mathrm{n}}\right\}$, interconnected by communication links and a set of $m$ tasks $T=\left\{t_{1}, t_{2}, \ldots \ldots . . t_{m}\right\}$. The processing efficiency of individual processor is given in the form of Execution Cost Matrix, $\operatorname{ECM}($,$) , of order \mathrm{m} x \mathrm{n}$ and Inter Task Communication Cost Matrix, ITCCM(,), of order $\mathrm{mx} \mathrm{m}$. This technique can generate an optimal solution by minimizing overall computational cost and allowing large number of computing tasks.

\subsection{Notations}

$\mathrm{T}$ : a set of tasks of a parallel program to be executed

$\mathrm{P}$ : the set of processors

$\mathrm{n}:$ the number of processors

$\mathrm{m}$ : total number of tasks that constitute the program.

$\operatorname{ECM}($,$) : Execution cost matrix of order \mathrm{m} \times \mathrm{n}$

$\operatorname{ITCCM}($,$) : Inter Task Communication Cost Matrix of order$ $\mathrm{m} \times \mathrm{m}$

$\operatorname{COSTEX}()$ : Array to store execution cost of clusters on processors.

$\operatorname{COSTCC}()$ : Array to store the communication cost of a cluster with all other clusters.

TOC : Total Optimal Cost

$\operatorname{ACL}($,$) : contains all the possible clusters that are formed.$

$\operatorname{SUM}($,$) : contains corresponding ECs of all the clusters that$ are in $\operatorname{ACL}($,$) .$

TPIN(,) : contains all the clusters that are finally selected from $\operatorname{ACL}($,$) .$

FIN(,) : contains corresponding ECs of the cluster stored in $\operatorname{TPIN}($,$) .$

$\operatorname{ALLOC}($,$) : contains the position of the processor on which a$ cluster is assigned.

$\mathrm{e}_{\mathrm{ij}}$ : EC of task $\mathrm{t}_{\mathrm{i}}$ on processor $\mathrm{p}_{\mathrm{j}}$. When any task is unable to get executed on any processor its execution cost is assumed to be infinity.

$\mathrm{cc}_{\mathrm{ik}}$ : ITCC between task $\mathrm{t}_{\mathrm{i}}$ and task $\mathrm{t}_{\mathrm{k}}$

\subsection{Definitions}

Execution cost (EC): The execution cost ' $\mathrm{e}_{\mathrm{ij}}$ ' (where $1 \leq \mathrm{i} \leq \mathrm{m}$ and $1 \leq \mathrm{j} \leq \mathrm{n}$ ) of a task $t_{\mathrm{i}}$, running on a processor $\mathrm{p}_{\mathrm{j}}$ is the amount of the total cost needed for the execution of $t_{i}$ on $p_{j}$.

Inter Task Communication Cost (ITCC): The Inter Task Communication Cost depends on the amount of data units exchanged between the tasks. If the interacting tasks $t_{i}$ and $t_{k}$ are assigned to different processors, the communication cost ' $\mathrm{cc}_{\mathrm{ik}}$ ' is incurred due to the data units exchanged between them during the execution.

Total Optimal Cost (TOC): The total optimal cost is the overall system cost incurred when tasks are executed on various processors.

\subsection{Assumptions}

The present technique is based on the following assumptions:

(i) Whenever two or more tasks are assigned to the same processor, the ITCC between them is assumed to be zero.

(ii) If a task is not executable on a certain processor, due to absence of some resources, the Execution Cost of that task on that processor is taken to be very large (infinite).

(iii) The completion of a program from computational point of view means that all related tasks have got executed.

(iv) Reassignment of task is not possible i.e. the allocation policy is static. 


\section{PROPOSED TASK ALLOCATION METHOD AND ALGORITHM}

Since it is assumed that $m>n$, implying that more than one task may be allocated to a processor at a time. So, a task is allocated to a processor in such a way that total system cost is minimized. This inspires us to form clusters of tasks to make them equal to the number of processors. The task clusters $\mathrm{C}_{\mathrm{i}}(\mathrm{i}=1,2, \ldots \ldots . \mathrm{n})$ having $\left(\mathrm{t}_{\mathrm{r} 1}, \mathrm{t}_{\mathrm{r} 2} \ldots \ldots \ldots \mathrm{t}_{\mathrm{rk}}\right)$ tasks are formed by making possible combination of tasks and adding the corresponding ECs. The cluster, having minimum EC, is selected and stored in final matrix, $\operatorname{FIN}($,$) . The process$ continues until number of clusters equals the number of processors. The remaining tasks are merged to the existing cluster having the maximum ITCC ' $\mathrm{cc}_{\mathrm{ij}}$ '. At last, Hungarian Algorithm is applied to the FIN(,) and optimum system cost is obtained.

Once the final task clusters are selected, for each task $t_{r 1}, t_{r 2}$ $\ldots \ldots \ldots \mathrm{t}_{\mathrm{rk}}\left(\mathrm{r}_{\mathrm{i}}, \mathrm{i}=1,2, \ldots \mathrm{k}\right)$ in cluster $\mathrm{C}_{\mathrm{i}}(\mathrm{i}=1,2, \ldots \ldots \mathrm{n})$, the same tasks is merged in ITCCM(,). The communication between all the tasks in the same cluster is considered as zero. The procedure for merging is achieved by adding $\mathrm{r}_{1}{ }^{\text {th }}, \mathrm{r}_{2}^{\text {th }}, \ldots \ldots \mathrm{r}_{\mathrm{k}}^{\text {th }}$ row on $r_{1}^{\text {th }}$ row and remove all the other rows. Similarly $\mathrm{r}_{1}^{\text {th }}, \mathrm{r}_{2}^{\text {th }}, \ldots \ldots . \mathrm{r}_{\mathrm{k}}^{\text {th }}$ column are merged on $\mathrm{r}_{1}^{\text {th }}$ column and all other columns are removed. The reduced $\operatorname{ITCCM}($,$) is stored$ in NTT(,).

\subsection{Proposed Algorithm}

\section{Step 1: Input $\boldsymbol{m}$ and $\boldsymbol{n}$.}

Step 2: Input ECM(,)and ITCCM

Step 3: Calculate the total number of tasks in a cluster

$$
k=\left|\_(m / n) \_\right|
$$

Step 3.1: Calculate total number of possible clusters, $\boldsymbol{c l}$

Step 3.2: Obtain total number of remaining task

$r \boldsymbol{m}=\boldsymbol{m} \bmod \boldsymbol{n}$

Step 4: Form clusters as follows:

Step 4.1: Task - clusters (say, C) are created by forming possible task combinations having $\mathrm{k}$ tasks in each cluster. The first cluster is formed by combining $1^{\text {st }}, 2^{\text {nd }}, 3^{\text {rd }} \ldots$...upto $\mathrm{k}^{\text {th }}$ task. These clusters are stored in ACL(,).

Step 4.2: For all tasks that are in cluster $\mathrm{C}$, calculate the sum of ECs, on each processor and store these values in $\operatorname{SUM}($,).

Step 5: Store SUM(,) to SUMNEW(,).

Step 5.1: Find the minimum EC in SUMNEW(,) and store it in $\min$.

Step 5.2: The cluster, corresponding to $\mathbf{m i n}$, is stored in TPIN(,) and the corresponding ECs from SUMNEW(,) are stored in $\operatorname{FIN}($,$) .$

Step 5.3: Remove all the rows from ACL(,) containing either of the tasks that are in cluster $\mathrm{C}$.

If the number of clusters in TPIN(,) is $\boldsymbol{n}$ and $\boldsymbol{r m}$ is zero then, go to Step 7.

Step 6: If $\boldsymbol{r m}$ is not equal to zero, then
Step 6.1: Find the remaining tasks by comparing the list consisting of all tasks, (say $\operatorname{REM}()$ ) with $\operatorname{TPIN}($,), which contains the list of tasks that are included in any of the cluster. Remove all the tasks from $\operatorname{REM}()$ that are present in TPIN(,). The leftover tasks in $\operatorname{REM}()$ are the remaining tasks.

Step 6.2: For each remaining task, its communication with each cluster is calculated and stored in $\operatorname{COM}()$.

Step 6.3:

maxcom $=\operatorname{Max}\{\operatorname{COM}(1), \operatorname{COM}(2), \ldots . \operatorname{COM}(\boldsymbol{n})\}$

Step 6.4: Merge the remaining task with the cluster which corresponds to maxcom

Step 6.4.1: Update FIN(,) and TPIN(,).

For load balancing no two remaining tasks are merged to the same cluster.

Step 7: According to the clusters, stored in $\operatorname{TPIN}($,$) , the$ corresponding tasks are merged in $\operatorname{ITCCM}($,$) and the result is$ stored NTT(,)

Step 8: Store $\mathrm{FIN}($,$) to \operatorname{COST}($,$) and apply Hungarian$ Algorithm [5] to FIN(,).

The positions of the allocations of clusters on the processors are saved in ALLOC(,)

Step 9: According to the positions stored in $\operatorname{ALLOC}($,$) , the$ ECs of each cluster is found from $\operatorname{COST}($,$) and result is taken$ in $\operatorname{COSTEX}()$

Step 10: Corresponding to each of the cluster the ITCC is found from $\operatorname{FITCCM}($,$) and results are stored in \operatorname{COSTCC}($,

Step 11:

$$
\begin{aligned}
& \operatorname{COE}=\sum_{\mathrm{i}=1}^{\mathrm{n}} \operatorname{COSTEX}(\mathrm{i}) \\
& \mathrm{COC}=\sum_{\mathrm{i}=1}^{\mathrm{n}} \operatorname{cosTcC}(\mathrm{i})
\end{aligned}
$$

Step 12: $\mathrm{TOC}=\mathrm{COE}+\mathrm{COC}$

Step 13: Stop.

\subsection{Implementation of Algorithm}

Example - 1 In this example, we have considered a typical program made up of 7 - executable tasks $\left\{\mathrm{t}_{1}, \mathrm{t}_{2}, \mathrm{t}_{3}, \mathrm{t}_{4}, \mathrm{t}_{5}, \mathrm{t}_{6}, \mathrm{t}_{7}\right\}$ to be executed on a DCS having three processors $\left\{\mathrm{p}_{1}, \mathrm{p}_{2}, \mathrm{p}_{3}\right\}$. The execution cost of each task on different processors and ITCC between the tasks is taken in the form of matrices $\operatorname{ECM}($,$) and \operatorname{ITCCM}($,$) respectively. This example deals with$ the more general situation where the case of remaining task(s) is taken care of. The processors connections are shown in Figure 1. The task execution and inter task communication graphs are illustrated in Figure 2 and Figure 3.

Step 1: Input $\boldsymbol{m}=7, \boldsymbol{n}=3$

Step 2: Input 


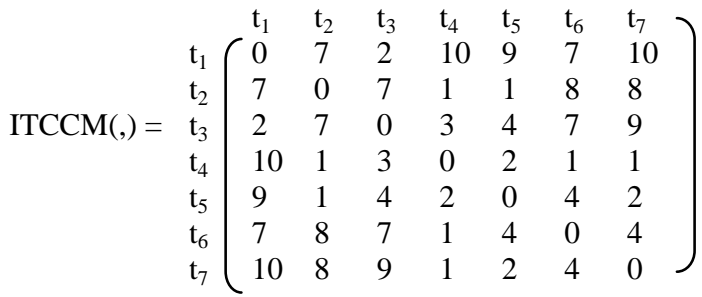

$$
\begin{aligned}
& \left.\operatorname{ECM}(,)=\begin{array}{cccc}
\mathrm{p}_{1} & \mathrm{p}_{2} & \mathrm{p}_{3} \\
\mathrm{t}_{1} & 12 & 18 & 15 \\
\mathrm{t}_{2} & 9 & 18 & 8 \\
\mathrm{t}_{3} & 19 & 7 & 15 \\
\mathrm{t}_{4} & 5 & 11 & 10 \\
\mathrm{t}_{5} & 12 & 17 & 5 \\
\mathrm{t}_{6} & 10 & 7 & 11 \\
\mathrm{t}_{7} & 17 & 2 & 12
\end{array}\right)
\end{aligned}
$$

Step 3: $k=2$

Step 3.1: $c l=21$

Step 3.2: $\mathrm{rm}=1$

Step 4:

$$
\operatorname{ACL}(,)=\left(\begin{array}{c}
\mathrm{t}_{1} \mathrm{t}_{2} \\
\mathrm{t}_{1} \mathrm{t}_{3} \\
\mathrm{t}_{1} \mathrm{t}_{4} \\
\mathrm{t}_{1} \mathrm{t}_{5} \\
\mathrm{t}_{1} \mathrm{t}_{6} \\
\mathrm{t}_{1} \mathrm{t}_{7} \\
\mathrm{t}_{2} \mathrm{t}_{3} \\
\mathrm{t}_{2} \mathrm{t}_{4} \\
\mathrm{t}_{2} \mathrm{t}_{5} \\
\mathrm{t}_{2} \mathrm{t}_{6} \\
\mathrm{t}_{2} \mathrm{t}_{7} \\
\mathrm{t}_{3} \mathrm{t}_{4} \\
\mathrm{t}_{3} \mathrm{t}_{5} \\
\mathrm{t}_{3} \mathrm{t}_{6} \\
\mathrm{t}_{3} \mathrm{t}_{7} \\
\mathrm{t}_{4} \mathrm{t}_{5} \\
\mathrm{t}_{4} \mathrm{t}_{6} \\
\mathrm{t}_{4} \mathrm{t}_{7} \\
\mathrm{t}_{5} \mathrm{t}_{6} \\
\mathrm{t}_{5} \mathrm{t}_{7} \\
\mathrm{t}_{6} \mathrm{t}_{7}
\end{array}\right) \quad \mathrm{SUM}(,)=\left(\begin{array}{lll}
\mathrm{p}_{1} & \mathrm{p}_{2} & \mathrm{p}_{2} \\
21 & 36 & 23 \\
31 & 25 & 30 \\
17 & 29 & 25 \\
24 & 35 & 20 \\
22 & 25 & 26 \\
29 & 20 & 27 \\
28 & 25 & 23 \\
14 & 29 & 18 \\
21 & 35 & 13 \\
19 & 25 & 19 \\
26 & 20 & 20 \\
24 & 18 & 25 \\
31 & 24 & 20 \\
29 & 14 & 26 \\
36 & 9 & 27 \\
17 & 28 & 15 \\
15 & 18 & 21 \\
22 & 13 & 22 \\
22 & 24 & 16 \\
29 & 19 & 17 \\
27 & 9 & 23
\end{array}\right)
$$

Step 5: $\operatorname{SUMNEW}()=,\operatorname{SUM}($,

Step 5.1: $\min =9$

Step 5.2:

$$
\begin{aligned}
& \operatorname{TPIN}(,)=\left[\begin{array}{ll}
\mathrm{t}_{3} & \mathrm{t}_{7}
\end{array}\right] \\
& \operatorname{FIN}(,)=\left[\begin{array}{ccc}
\mathrm{p}_{1} & \mathrm{p}_{2} & \mathrm{p}_{3} \\
36 & 9 & 27
\end{array}\right]
\end{aligned}
$$

Step 5.3: Remove the cluster that consists of either $t_{3}$ or $t_{7}$ from ACL(,) and corresponding ECs from SUMNEW(,). Now, the ACL(,) and $\operatorname{SUMNEW}($,$) are:$

$\operatorname{ACL}()=,\left(\begin{array}{c}t_{1} t_{2} \\ t_{1} t_{4} \\ t_{1} t_{5} \\ t_{1} t_{6} \\ t_{2} t_{4} \\ t_{2} t_{5} \\ t_{2} t_{6} \\ t_{2} t_{7} \\ t_{4} t_{5} \\ t_{4} t_{6} \\ t_{5} t_{6} \\ \end{array}\right)$ SUMNEW(,) $=\left(\begin{array}{ccc}p_{1} & p_{2} & p_{2} \\ 21 & 36 & 23 \\ 17 & 29 & 25 \\ 24 & 35 & 20 \\ 22 & 25 & 26 \\ 14 & 29 & 18 \\ 21 & 35 & 13 \\ 19 & 25 & 19 \\ 26 & 20 & 20 \\ 17 & 28 & 15 \\ 15 & 18 & 21 \\ 22 & 24 & 16 \\ & & \end{array}\right)$

Again select the next minimum value, i.e. 13 from the remaining rows of $A C L($,$) and corresponding cluster t_{2} t_{5}$ from SUMNEW(,). So, TPIN(,) and FIN(,) become:

$$
\begin{aligned}
\operatorname{TPIN}(,) & =\left[\begin{array}{l}
\mathrm{t}_{3} \mathrm{t}_{7} \\
\mathrm{t}_{2} \mathrm{t}_{5}
\end{array}\right] \\
\operatorname{FIN}(,) & =\left[\begin{array}{ccc}
\mathrm{p}_{1} & \mathrm{p}_{2} & \mathrm{p}_{3} \\
21 & 9 & 27 \\
21 & 35 & 13
\end{array}\right]
\end{aligned}
$$

Remove the clusters that either contains task $t_{2}$ or $t_{5}$ from $\operatorname{ACL}($,$) and their corresponding ECs from SUMNEW(,).$ Now, the ACL(,), SUMNEW(,) are:

$$
\begin{gathered}
\operatorname{ACL}(,)=\left[\begin{array}{l}
\mathrm{t}_{1} \mathrm{t}_{4} \\
\mathrm{t}_{4} \mathrm{t}_{6}
\end{array}\right] \\
\operatorname{SUMNEW}(,)=\left[\begin{array}{lll}
17 & \mathrm{p}_{2} & \mathrm{p}_{3} \\
15 & 18 & 29
\end{array}\right]
\end{gathered}
$$

Again, select the next minimum value, i.e. 15 from the remaining rows of $\operatorname{ACL}($,$) and corresponding cluster \mathrm{t}_{4} \mathrm{t}_{6}$ from SUMNEW(,) resulting into $\operatorname{TPIN}($,$) and \operatorname{FIN}($,$) as$

$$
\operatorname{TPIN}(,)=\left[\begin{array}{l}
\mathrm{t}_{3} \mathrm{t}_{7} \\
\mathrm{t}_{2} \mathrm{t}_{5} \\
\mathrm{t}_{4} \mathrm{t}_{6}
\end{array}\right]
$$

$$
\mathrm{FIN}(,)=\left[\begin{array}{ccc}
\mathrm{p}_{1} & \mathrm{p}_{2} & \mathrm{p}_{3} \\
36 & 9 & 27 \\
21 & 35 & 13 \\
15 & 18 & 21
\end{array}\right]
$$

Here, the number of clusters in TPIN(,) is 3 and $\boldsymbol{r m}$ is not zero hence,

Step 6: $\quad r m=1$

Step 6.1: $\operatorname{REM}()=\mathrm{t}_{1}$ 
Step 6.2: The communication cost of $t_{1}$ is calculated with each cluster as:

$$
\begin{aligned}
& \operatorname{COM}(1)=12 \\
& \operatorname{COM}(2)=16 \\
& \operatorname{COM}(3)=17
\end{aligned}
$$

Step 6.3: maxcom $=17$

Step 6.4: So, the third cluster, having tasks $\mathrm{t}_{4} \mathrm{t}_{6}$, is selected for final merging resulting in

$$
\begin{aligned}
\operatorname{TPIN}(,) & =\left[\begin{array}{l}
\mathrm{t}_{3} \mathrm{t}_{7} \\
\mathrm{t}_{2} \mathrm{t}_{5} \\
\mathrm{t}_{4} \mathrm{t}_{6} \mathrm{t}_{1}
\end{array}\right] \\
\operatorname{FIN}(,) & =\left[\begin{array}{lll}
\mathrm{p}_{1} & \mathrm{p}_{2} & \mathrm{p}_{3} \\
26 & 9 & 27 \\
21 & 35 & 13 \\
27 & 36 & 36
\end{array}\right]
\end{aligned}
$$

Step 7:

$$
\operatorname{ITCCM}(,)=\begin{array}{lll}
\mathrm{t}_{3} \mathrm{t}_{7} & \mathrm{t}_{2} \mathrm{t}_{5} & \mathrm{t}_{4} \mathrm{t}_{6} \mathrm{t}_{1} \\
\mathrm{t}_{2} \mathrm{t}_{5} & \mathrm{t}_{1} \mathrm{t}_{4} \mathrm{t}_{6}
\end{array}\left[\begin{array}{lll}
21 & 0 & 27 \\
31 & 27 & 0
\end{array}\right]
$$

$\operatorname{NTT}()=,\operatorname{ITCCM}()$

Step 8: $\operatorname{COST}()=,\mathrm{FIN}($,). On applying Hungarian Algorithm [5] to the FIN (,). We get

$$
\operatorname{ALLOC}(,)=\left[\begin{array}{ccc}
0 & 1 & 0 \\
0 & 0 & 1 \\
1 & 0 & 0
\end{array}\right]
$$

The allocations obtained after implementing the row and column assignment processes is shown in Table 1.

Step 9: $\operatorname{COSTEX}=(9,13,27)$

Step 10: $\operatorname{COSTCC}=(21,31,27)$

$$
\text { Step 11: } \begin{aligned}
\mathrm{COE}=9+ & 13+27 \\
& =49 \\
\mathrm{COC}= & 21+31+27 \\
& =79
\end{aligned}
$$

Step 12: $\mathrm{TOC}=\mathrm{COE}+\mathrm{COC}$

$$
=49+79=128 \text {. }
$$

Step 13: Stop.

The optimal assignment graph is shown in Figure 4. The performance comparison of proposed algorithm and the algorithm discussed in [5] on the same data set is shown in Table 2.

\section{RESULTS AND CONCLUSIONS}

In this paper, the problem of task allocation, considering load balancing using task clustering, is discussed. As the task allocation problem is known to be NP- hard, the proposed technique finds near optimal system cost. Static load balancing task allocation policy is used to achieve this objective. The proposed algorithm tries to form clusters of tasks and then allocate these clusters to the processors. The effectiveness of the proposed algorithm is compared with the static version of the algorithm proposed in [5]. For several sets of input data $(m, n)$, the comparison is shown in tabular form as well as in graphical form. The Table 3 and Table 4 illustrated in the form of Figure 5 and Figure 6 respectively.

It can be observed from Figure 5 that the values of total optimal cost obtained by the present algorithm are less as compared to those obtained in [5], in the case, when the number of processors is kept fixed and number of tasks are taken in increasing order. The similar observation can also be made from Figure 6 in the case when the number of tasks is fixed and number of processors is taken in increasing order. Thus, it is concluded that the present algorithm results into better optimal cost in both the cases.

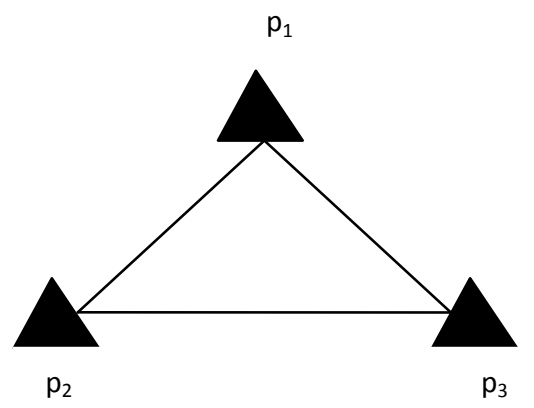

Figure 1: Processors Connections of Example 1 


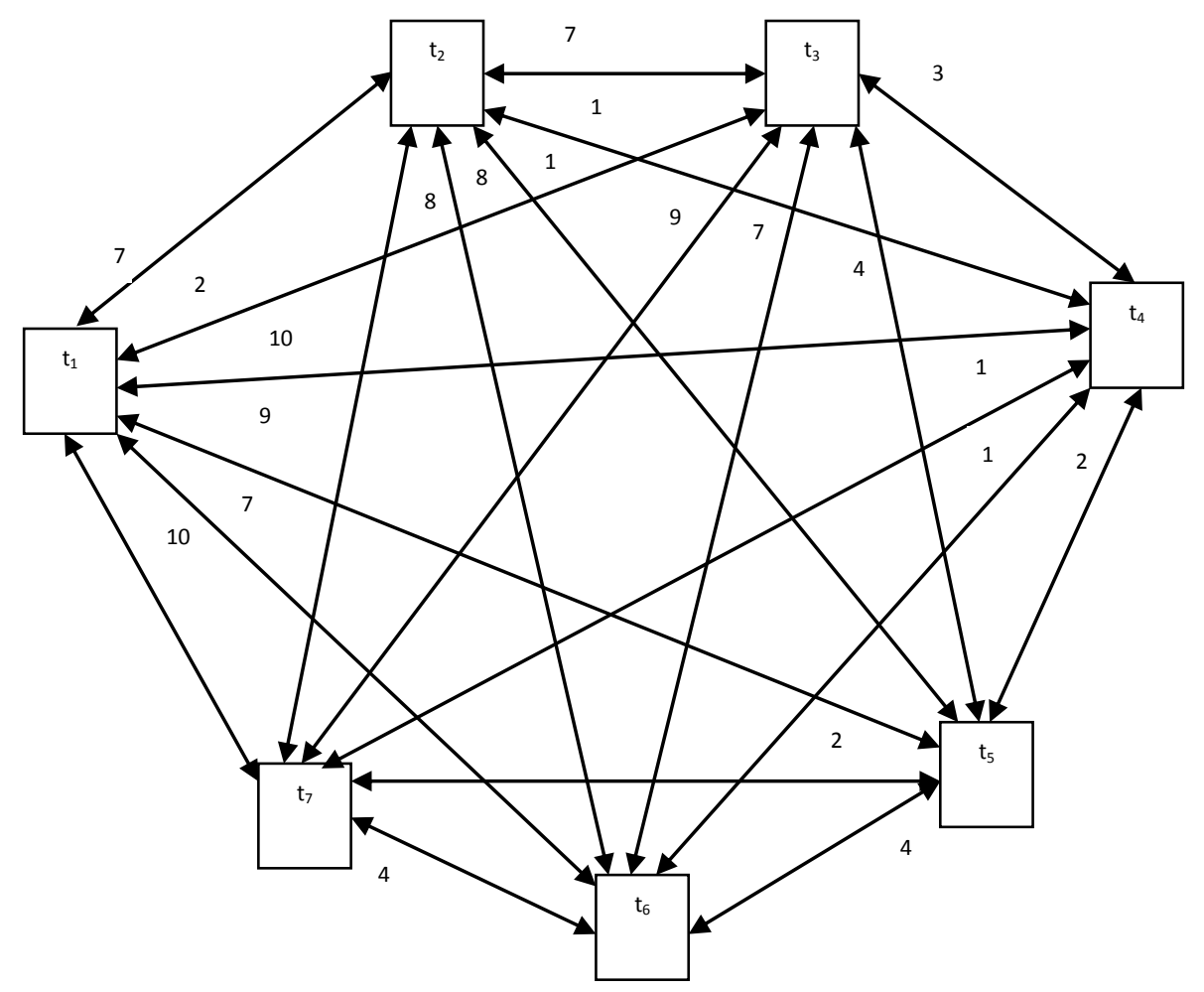

Figure 2: Inter Task Communication Graph of Example 1

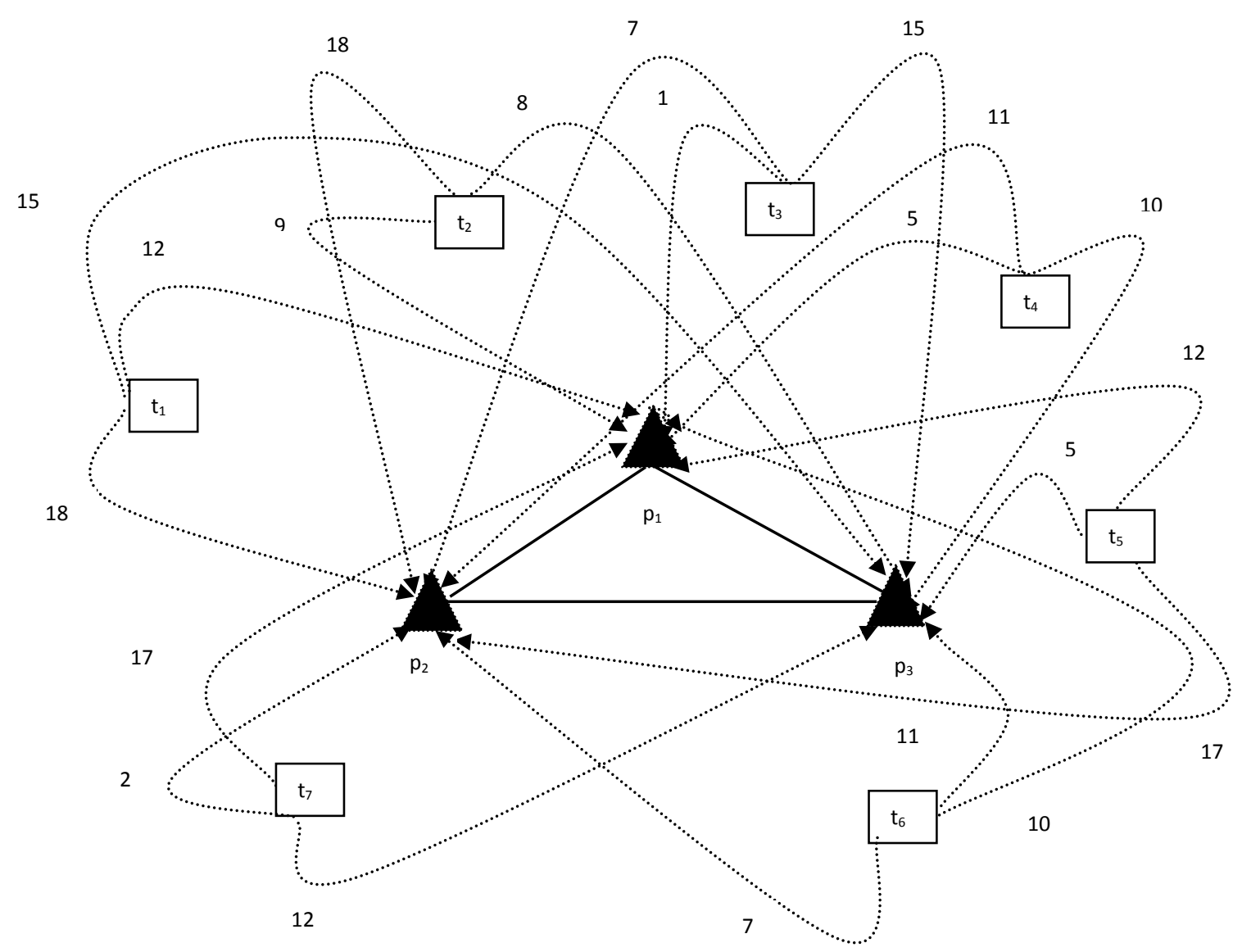

Figure 3: Task Execution Graph of Example 1 


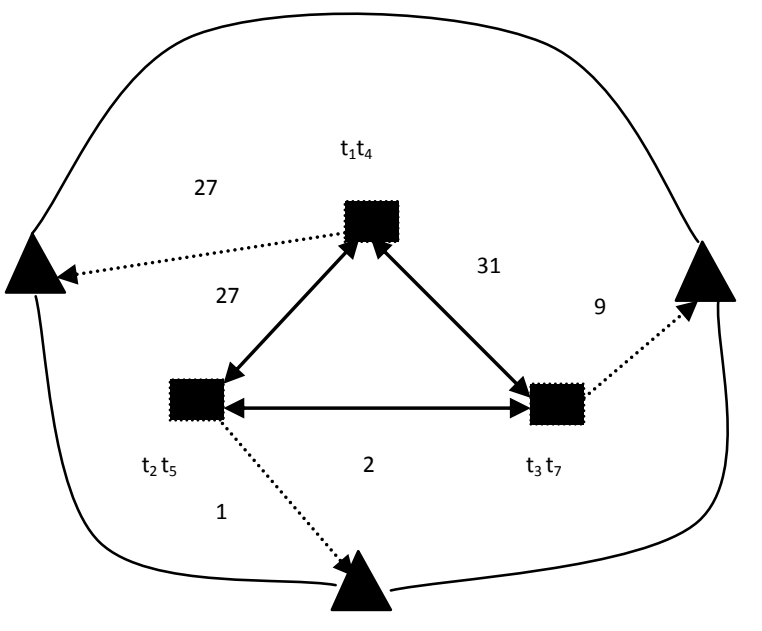

Figure 4: Optimal Assignment Graph of Example 1

Table 1: Optimal Allocation Table of Example 1

\begin{tabular}{|l|l|l|}
\hline Tasks & Processors & EC \\
\hline $\mathrm{t}_{1} \mathrm{t}_{4} \mathrm{t}_{6}$ & $\mathrm{p}_{1}$ & 27 \\
\hline $\mathrm{t}_{2} \mathrm{t}_{5}$ & $\mathrm{p}_{3}$ & 13 \\
\hline $\mathrm{t}_{3} \mathrm{t}_{7}$ & $\mathrm{p}_{2}$ & 9 \\
\hline
\end{tabular}

Table 2: Comparison of Results for Example 1

\begin{tabular}{|c|c|c|}
\hline $\begin{array}{l}\text { Model } \rightarrow \\
\text { Result } \downarrow\end{array}$ & $\begin{array}{l}\text { Proposed } \\
\text { Algorithm }\end{array}$ & $\begin{array}{c}5] \\
\text { Algorithm }\end{array}$ \\
\hline ALLOCATIONS & $\begin{array}{l}\mathrm{t}_{1} \mathrm{t}_{4} \mathrm{t}_{6} \rightarrow \mathrm{p}_{1} \\
\mathrm{t}_{2} \mathrm{t}_{5} \rightarrow \mathrm{p}_{3} \\
\mathrm{t}_{3} \mathrm{t}_{7} \rightarrow \mathrm{p}_{2}\end{array}$ & $\begin{array}{l}\mathrm{t}_{1} \mathrm{t}_{3} \mathrm{t}_{7} \rightarrow \mathrm{p}_{2} \\
\mathrm{t}_{2} \mathrm{t}_{6} \rightarrow \mathrm{p}_{1} \\
\mathrm{t}_{4} \mathrm{t}_{5} \rightarrow \mathrm{p}_{3}\end{array}$ \\
\hline COSTEX & 49 & 61 \\
\hline COSTCC & 79 & 76 \\
\hline TOC & 128 & 137 \\
\hline
\end{tabular}

Table 3: Comparison of TOC When Number of Tasks is in Increasing Order

\begin{tabular}{|c|c|c|c|}
\hline $\begin{array}{c}\text { Tasks } \\
\mathbf{m}\end{array}$ & $\begin{array}{c}\text { Processors } \\
\mathbf{n}\end{array}$ & $\begin{array}{c}\text { Proposed } \\
\text { Algorithm }\end{array}$ & $\begin{array}{c}\text { [5] } \\
\text { Algorithm }\end{array}$ \\
\hline 6 & 4 & 104.8 & 110.7 \\
\hline 7 & 4 & 138.2 & 142.5 \\
\hline 8 & 4 & 178.2 & 184 \\
\hline 9 & 4 & 217 & 226.6 \\
\hline 10 & 4 & 238.1 & 257.8 \\
\hline 11 & 4 & 316.7 & 333.8 \\
\hline 12 & 4 & 366.3 & 378.1 \\
\hline 13 & 4 & 394.1 & 423.4 \\
\hline
\end{tabular}

Table 4: Comparison of TOC When Number of Processors is in Increasing Order

\begin{tabular}{|c|c|c|c|}
\hline $\begin{array}{c}\text { Tasks } \\
\mathbf{m}\end{array}$ & $\begin{array}{c}\text { Processors } \\
\mathbf{n}\end{array}$ & $\begin{array}{c}\text { Proposed } \\
\text { Algorithm }\end{array}$ & $\begin{array}{c}\text { [5] } \\
\text { Algorithm }\end{array}$ \\
\hline 13 & 4 & 420.9 & 443.1 \\
\hline 13 & 5 & 443.2 & 475.6 \\
\hline 13 & 6 & 440 & 468.5 \\
\hline 13 & 7 & 451.8 & 473.5 \\
\hline 13 & 8 & 450.2 & 475.8 \\
\hline 13 & 9 & 446.5 & 470.9 \\
\hline 13 & 10 & 457 & 497.3 \\
\hline 13 & 11 & 461.1 & 491.5 \\
\hline 13 & 12 & 453.2 & 475 \\
\hline
\end{tabular}




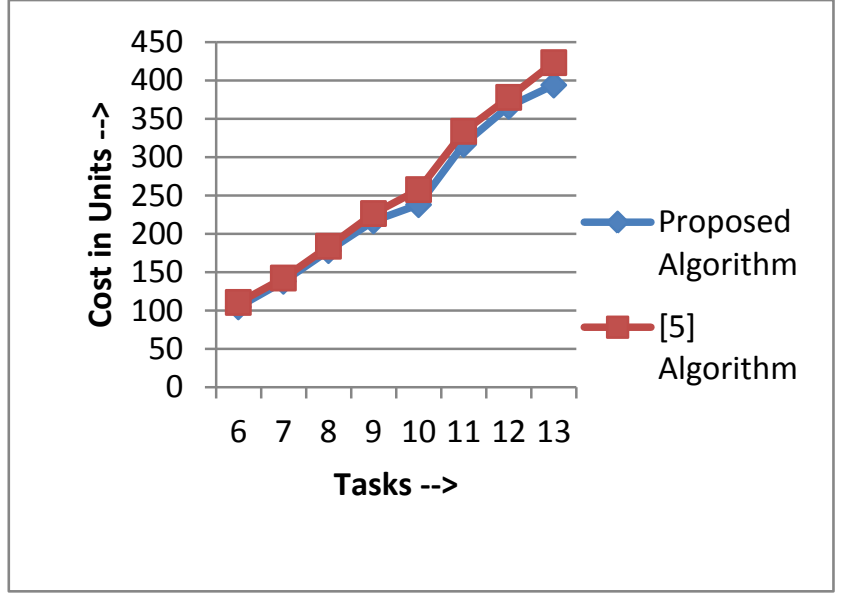

Figure 5: TOC When Tasks are in an Increasing Order and Number of Processors is 4

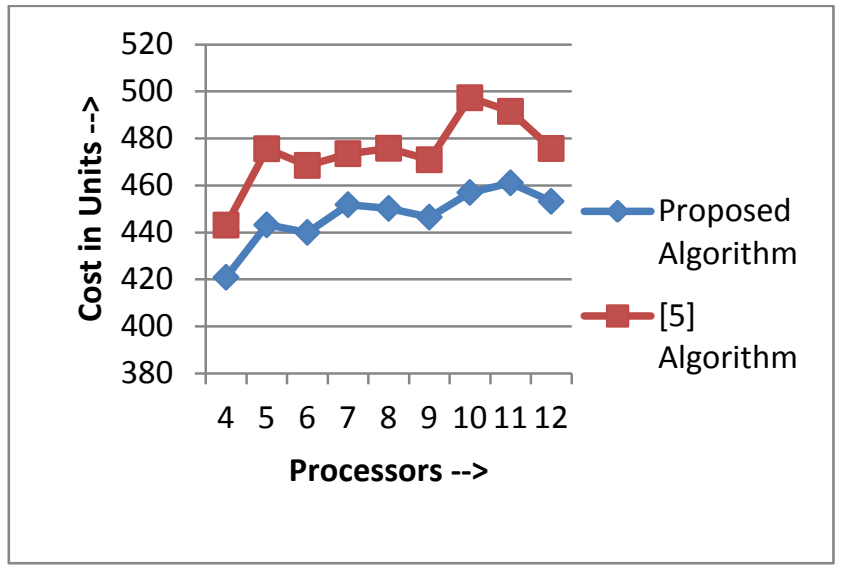

Figure 6: TOC When Processors are in an Increasing Order and Number of Tasks is 13

\section{REFERENCES}

[1] Alakeel Ali M. 2010. A guide to dynamic load balancing in distributed computer systems. International Journal of Computer Science and Network Security, 10 (6), 153 -160.

[2] Bokhari, S. H. 1987. Assignment problems in parallel and distributed computing.

[3] Chu, W. W., Holloway, L. J., Lan, M.T., and Efe, K. 1980. Task allocation in distributed data processing. IEEE Computer, 13(11) (Nov. 1980), 57-69.

[4] Gerasoulis, A. and Yang, T. A. 1992. A comparison of clustering heuristics for scheduling directed acyclic graphs on multiprocessors. Journal of Parallel and Distributed Computing, (1992), 276 - 291.

[5] Govil, Kapil 2011. A smart algorithm for dynamic task allocation for distributed processing environment. International Journal of Computer Applications, 28(2), (2011), 13-19.

[6] Gillett, Billy E. 1979. Introduction to operations research: a computer-oriented algorithmic approach.
[7] Kafil, M., and Ahmad, I. 1998. Optimal Task Assignment in Heterogeneous Computing Systems. IEEE Concurrency, (1998), 42- 51.

[8] Kim, S. J., and Browne, J. C. 1988. A general approach to mapping of parallel computations upon multiprocessor architectures. In Proceedings of the International Conference on Parallel Processing.

[9] Kumar, Avanish, Sharma, Abhilasha and Dhagat, Vanita Ben 2010. Maximal link mode algorithm for task allocation in distributed computing systems. Proceedings of the $4^{\text {th }}$ National Conference: INDIACom-2010.

[10]Liou, J. -C. and Palis, M. A. 1996. An efficient clustering heuristic for scheduling dags on multiprocessor. In Proceedings of the $8^{\text {th }}$ Symposium on Parallel and Distributed Processing.

[11] Palis M. A., Liou, J. -C. and Wei, D. S. 1996. Task clustering and scheduling for distributed memory parallel architectures. IEEE Transactions on Parallel and Distributed Systems (1996), 46 - 55.

[12] Raii, Anurag and Kapoor, Vikram 2012. Efficient clustering model for utilization of processor's capacity in distributed computing system. International Journal of Computer Applications, 44(23), 21- 25.

[13] Richard, R.Y., Lee, E.Y.S. and Tsuchiya, M. 1982. A task allocation modal for distributed computer system. IEEE Transaction on Computers 31, 41- 47.

[14] Sagar, G. and Sarje, A.K. 1991. Task allocation modal for distributed system. International Journal of System Science, 22(9), 1671 - 1678

[15] Sarje, A. K. and Sagar, G. 1991. Heuristic modal for task allocation in distributed computer systems. IEEE Proceedings on Computers and Digital Techniques, $138(5), 313-318$.

[16] Saxena Pankaj, Govil Kapil, Saxena Gaurav, Kumar Saurabh and Agrawal Neha. 2012. An algorithmic approach and comparative analysis of task assignment to processor for achieving time efficiency in process completion. International Journal of Applied Engineering and Technology, 2(1), 114-119.

[17] Sharma, Manisha, Kumar, Harendra, Garg, Deepak. 2012. An Optimal Task Allocation Model through Clustering with Inter-Processor Distances in Heterogeneous Distributed Computing Systems. International Journal of Soft Computing and Engineering, 2(1), 50- 55.

[18] Srinivasan, S., and Jha, N. K. 1999. Safety and reliability driven task allocation in distributed systems. IEEE Transactions on Parallel and Distributed Systems. 10(3), $238-251$

[19] Vidyarthi, Deo Prakash, Tripathi, Anil Kumar, Sarker, Biplab Kumer, Dhawan, Abhishek and Yang, Laurence Tianruo. 2004. Cluster - based multiple task allocation in distributed computing system. In Proceedings of the $18^{\text {th }}$ International Parallel and Distributed Processing Symposium (IPDPS'04).

[20] Yang, T. and Gerasoulis, A. 1994. DSC: scheduling parallel tasks on an unbounded number of processors. IEEE Transactions on Parallel and Distributed Systems. 5(9), 951- 967 